\title{
Geometric dissipation in kinetic equations
}

\author{
Darryl D. Holm ${ }^{\mathrm{a}}$, Vakhtang Putkaradze ${ }^{\mathrm{b}}$, Cesare Tronci ${ }^{\mathrm{c}}$ \\ ${ }^{a}$ Department of Mathematics, Imperial College London, London SW7 2AZ, UK \\ Computer and Computational Science Division, Los Alamos National Laboratory, Los Alamos, NM, 87545 USA \\ ${ }^{\mathrm{b}}$ Department of Mathematics, Colorado State University, Fort Collins, CO 80523 USA \\ Institute for Theoretical Physics, Universität Köln, Zuplicher Str. 77, 50968 Köln, Germany \\ ${ }^{\mathrm{c}}$ Department of Mathematics, Imperial College London, London SW7 2AZ, UK \\ TERA Foundation for Oncological Hadrontherapy, 11 V. Puccini, Novara 28100, Italy
}

\begin{abstract}
A new symplectic variational approach is developed for modeling dissipation in kinetic equations. This approach yields a double bracket structure in phase space which generates kinetic equations representing coadjoint motion under canonical transformations. The Vlasov example admits measure-valued single-particle solutions. Such solutions are reversible. The total entropy is a Casimir, and thus it is preserved.

To cite this article: D.D.Holm, V. Putkaradze and C. Tronci, C. R. Acad. Sci. Paris, Ser. I XXX (2007).

\section{Résumé}

Une nouvelle approche est proposée pour modeliser la dissipation dans les équations cinétiques. Cette approche produit une structure à double crochet dans l'espace des phases qui conduit aux équations cinétiques d'une dynamique coadjointe après transformations canoniques. L'exemple de Vlasov admet alors des solutions pour particule unique. Ces solutions sont réversibles; l'entropie totale est un Casimir et est donc préservée.

Pour citer cet article :D.D.Holm, V. Putkaradze and C. Tronci, C. R. Acad. Sci. Paris, Ser. I XXX (2007).
\end{abstract}

Version française abrégée Une nouvelle approche est proposée pour la modélisation des phénomènes dissipatifs dans les équations cinétiques [4,18]. Cette construction est réalisée de telle sorte que la géométrie de la variable dynamique est préservée : en particulier, nous considérons l'équation de Vlasov [18] comme

Email addresses: d.holm@imperial.ac.uk (Darryl D. Holm), putkarad@math.colostate.edu (Vakhtang Putkaradze), cesare.tronci@imperial.ac.uk (Cesare Tronci). 
modèle naturel de conservation dans l'espace des phases. De plus nous introduisons une quantité particulière nommée "mobilité", inspirée par analogie avec la loi de Darcy pour systèmes continus avec auto-agrégation $[9,10,11]$. Dans ce cas, on introduit la mobilité comme le facteur de proportionnalité entre la force agissant sur les particules et leur vitesse. Nous nous intéressons ainsi à une forme de dissipation qui peut généraliser les phénomènes d'auto-agrégation aux systèmes cinétiques dans l'espace des phases.

Une telle approche produit une structure à double crochet [1] dans l'espace des phases similaire à celle présentée dans la littérature pour la modélisation de certains systèmes astrophysiques [13]. Cette structure génère une dynamique coadjointe réversible (Éq. 2) via l'action des transformations canoniques. On trouve finalement que toutes les fonctionnelles de la distribution sont de type Casimir et que l'entropie est préservée (Proposition 2.2).

L'innovation de notre approche se voit dans le rôle que la mobilité peut jouer comme opération de filtre (o moyenne) sur la fonction de distribution des particules. Par conséquent on définit la mobilité comme une fonctionnelle de la distribution des particules. Ce fait conduit à l'existence de la solution de particule unique qui n'est pas présente dans les anciennes approches et représente le résultat principal de cet article (Théorème 4.1).

\section{Introduction}

Non-linear dissipation in physical systems can modeled by the sequential application of two Poisson brackets, just as in magnetization dynamics [8]. A similar double bracket operation for modeling dissipation has been proposed for the Vlasov equation. Namely,

$$
\frac{\partial f}{\partial t}+\left[f, \frac{\delta H}{\delta f}\right]=\alpha\left[f,\left[f, \frac{\delta H}{\delta f}\right]\right],
$$

where $\alpha>0$ is a positive constant, $H$ is the Vlasov Hamiltonian and $[\cdot, \cdot]$ is the canonical Poisson bracket. When $\alpha \rightarrow 0$, this equation reduces the Vlasov equation for collisionless plasmas. For $\alpha>0$, this is the double bracket dissipation approach for the Vlasov-Poisson equation introduced in Kandrup [13] and developed in Bloch et al. [1]. This double bracket approach for introducing dissipation into the Vlasov equation differs from the standard Fokker-Planck linear diffusive approach [4], which adds dissipation on the right hand side as the Laplace operator in the momentum coordinate $\Delta_{p} f$.

An interesting feature of the double bracket approach is that the resulting symmetric bracket gives rise to a metric tensor and an associated Riemannian (rather than symplectic) geometry for the solutions. The variational approach also preserves the advective nature of the evolution of Vlasov phase space density, by coadjoint motion under the action of the canonical transformations on phase space densities.

As Otto [17] explained, the geometry of disspation may be understood as emerging from a variational principle. Here, we apply the variational approach to derive the following generalization of the double bracket structure in equation (1) that recovers previous cases for particular choices of modeling quantities,

$$
\frac{\partial f}{\partial t}+\left[f, \frac{\delta H}{\delta f}\right]=\left[f,\left[\mu(f), \frac{\delta E}{\delta f}\right]\right] .
$$

Eq. (2) extends the double bracket operation in (1) and reduces to it when $H$ is identical to $E$ and $\mu(f)=\alpha f$. The form (2) of the Vlasov equation with dissipation allows for more general mobilities than those in $[1,13,14,16]$. For example, one may choose $\mu[f]=K * f$ (in which $*$ denotes convolution in phase space). As in [12] the smoothing operation in the definition of $\mu(f)$ introduces a fundamental length scale

(the filter width) into the dissipation mechanism. Smoothing has the added advantage of endowing (2) with the one-particle solution as its singular solution. The generalization Eq. (2) may also be justified by 
using thermodynamic and geometric arguments [12]. In particular, this generalization extends the classic Darcy's law (velocity being proportional to force) to allow the corresponding modeling at the microscopic statistical level.

\section{Dissipation for kinetic equations}

We aim to model dissipation in Vlasov kinetic systems through a suitable generalization of Darcy's law. Indeed, we believe that the basic ideas of Darcy's Law in configuration space can be transferred to a phase space treatment giving rise to the kinetic description of self-organizing collisionless multiparticle systems. In what follows, we will construct kinetic equations for geometric order parameters that respect the symplectic nature of the phase space by considering the Lie algebra of generating functions of canonical transformations (symplectomorphisms).

The first step is to establish how a geometric quantity evolves in phase space, so that the symplectic nature of its evolution is preserved. For this, we regard the action of the symplectic algebra as an action of the generating functions $h$ of $\kappa$, rather than vector fields. The action is formally expressed as $\kappa h=$ $£_{X_{h}} \kappa$. The dual operation of the action which we denote by $\star$ is then defined as $\langle\kappa \star \zeta, h\rangle=\left\langle\kappa,-\mathcal{L}_{X_{h}} \zeta\right\rangle$. Here $X_{h}(q, p)$ is the Hamiltonian vector field generated by a Hamiltonian function $h(q, p)$ through the definition $X_{h} \downarrow \omega:=d h$. Notice that the star operation takes values in the space $\mathcal{F}^{*}$ of phase space densities $\kappa \star \zeta \in \mathcal{F}^{*}$. In the particular case of interest here, $\kappa$ is the phase space density $\kappa=f d q \wedge d p$ and $\zeta=g$, a function on phase space. In this case, the star operation is simply minus the canonical Poisson bracket, $\kappa \star g=[f, g] d q \wedge d p$.

We shall first employ these considerations to find the purely dissipative part of the kinetic equation for a particle density on phase space. We choose variations of the form $\delta f=-£_{X_{h}(\phi)} \mu(f)=-[\mu(f), h(\phi)]$ with $h(\phi)=(\phi \star f)^{\sharp}=[\phi, f]$ where $(\cdot)^{\sharp}$ in $(f \star \phi)^{\sharp}$ transforms a phase space density into a scalar function. The operation $(\cdot)^{\sharp}$ will be understood in the pairing below. We then follow the steps:

$$
\left\langle\phi, \frac{\partial f}{\partial t}\right\rangle=\left\langle\frac{\delta E}{\delta f}, \delta f\right\rangle=\left\langle\frac{\delta E}{\delta f},-[\mu(f), h(\phi)]\right\rangle=\left\langle\left[\mu(f), \frac{\delta E}{\delta f}\right],[\phi, f]\right\rangle=\left\langle\phi,\left[f,\left[\mu(f), \frac{\delta E}{\delta f}\right]\right]\right\rangle .
$$

Therefore, a functional $F(f)$ satisfies the following evolution equation in bracket notation,

$$
\frac{d F}{d t}=\left\langle\frac{\partial f}{\partial t}, \frac{\delta F}{\delta f}\right\rangle=-\left\langle\left[\mu(f), \frac{\delta E}{\delta f}\right],\left[f, \frac{\delta F}{\delta f}\right]\right\rangle=:\{\{E, F\}\} .
$$

The mobility $\mu$ and dissipation energy functional $E$ appearing in (3) are modeling choices and must be selected based on the additional input from physics. The bracket (3) reduces to Kandrup's dissipative bracket for the modeling choice of $\mu(f)=\alpha f$ with some $\alpha>0$ [13]. The dissipation energy $E$ in Kandrup's paper was taken to be the Vlasov Hamiltonian (see below), but in our approach it also may be taken as a modeling choice. This extra freedom allows for more flexible interpretation and treatment of the dissipation process.

Proposition 2.1 There exist choices of mobility $\mu[f]$ for which the bracket (3) dissipates energy E. Proof. The dissipative bracket in equation (3) yields $\dot{E}=\{\{E, E\}\}$ which is negative when $\mu[f]$ is chosen appropriately. For example, $\mu[f]=f M[f]$, where $M[f] \geq 0$ is a non-negative scalar functional of $f$. (That is, $M[f]$ is a number.)

Remark 1 The dissipative bracket (3) satisfies the Leibnitz rule for the derivative of a product of functionals. In addition, it allows one to reformulate the equation (2) in terms of flow on a Riemannian manifold with a metric defined through the dissipation bracket, as discussed in more detail in [11]. 
Proposition 2.2 (Casimir functionals) For an arbitrary smooth function $\Phi$ the functional $C_{\Phi}=$ $\int \Phi(f) d q \wedge d p$ is preserved for any energy functional $E$.

Proof. It suffices to calculate the bracket

$$
\frac{d C_{\Phi}}{d t}=\left\{\left\{C_{\Phi}, E\right\}\right\}:=-\left\langle\left[\mu(f), \frac{\delta E}{\delta f}\right],\left[f, \frac{\delta C_{\Phi}}{\delta f}\right]\right\rangle=-\left\langle\left[\mu(f), \frac{\delta E}{\delta f}\right],\left[f, \Phi^{\prime}(f)\right]\right\rangle=0 .
$$

Corollary 2.3 The entropy functional $S=\int f \log f$ is preserved for any energy functional $E$.

Remark 2 The existence of Casimirs and the corresponding preservation of any entropy defined solely in terms of $f$ arises because the dissipative bracket (3) generates coadjoint motion, which is reversible. This property is shared with Kandrup's bracket, which is recovered for $\mu(f)=\alpha f$ for constant $\alpha>0$.

\section{Dissipation for kinetic moments: the Kupershmidt-Manin bracket and Darcy's law}

In this section we show how Eq. (2) leads very naturally to a nonlocal form of Darcy's law. In order to show how this equation is recovered, we introduce the Lie-Poisson structure for kinetic moments, also known as Kupershmidt-Manin structure [15]. We proceed by considering a one-dimensional configuration space; an extension to higher dimensions would also be possible by considering the treatment in [6].

As a general result $[5,6,7]$, the equations for the moments of the Vlasov equation are a Lie-Poisson system. The $n$-th moment is defined as

$$
A_{n}(q):=\int p^{n} f(q, p) d p .
$$

and the dynamics of these quantities is regulated by the Kupershmidt-Manin structure

$$
\{F, G\}=\left\langle A_{m+n-1}, \llbracket \frac{\delta F}{\delta A_{n}}, \frac{\delta G}{\delta A_{m}} \rrbracket\right\rangle,
$$

where summation over repeated indices is omitted and the Lie bracket $\llbracket \cdot, \cdot \rrbracket$ is defined as

$$
\llbracket \alpha_{m}, \beta_{n} \rrbracket=n \beta_{n}(q) \alpha_{m}^{\prime}(q)-m \alpha_{m}(q) \beta_{n}^{\prime}(q)=: \operatorname{ad}_{\alpha_{m}} \beta_{n}
$$

The moment equations are

$$
\dot{A}_{n}=-\operatorname{ad}_{\beta_{n}}^{*} A_{m+n-1}=-(n+m) A_{n+m-1} \frac{\partial \beta_{n}}{\partial q}-n \beta_{n} \frac{\partial A_{n+m-1}}{\partial q},
$$

where the $\mathrm{ad}^{*}$ operator is defined by $\left\langle\operatorname{ad}_{\beta_{n}}^{*} A_{k}, \alpha_{k-n+1}\right\rangle:=\left\langle A_{k}, \operatorname{ad}_{\beta_{n}} \alpha_{k-n+1}\right\rangle$.

At this point one can consider the following Lie algebra action on Vlasov densities

$$
\beta_{n} f:=£_{X_{p^{n} \beta_{n}}} f=\left[f, p^{n} \beta_{n}\right] \quad \text { (no sum) }
$$

which is obviously given by the action of the Hamiltonian function $h(q, p)=p^{n} \beta_{n}(q)$. Now, the dual action is given by

$$
\left\langle f \star_{n} g, \beta_{n}\right\rangle:=\left\langle f, \beta_{n} g\right\rangle=\left\langle f \star g, p^{n} \beta_{n}(q)\right\rangle=\left\langle\int\{f, g\} p^{n} d p, \beta_{n}\right\rangle
$$

and the dissipative bracket for the moments (3) is written in this notation as

$$
\begin{aligned}
\{\{E, F\}\} & =-\left\langle\int p^{n}\left[\mu[f], \frac{\delta E}{\delta f}\right] d p, \int p^{n}\left[f, \frac{\delta F}{\delta f}\right] d p\right\rangle \\
& =-\left\langle\operatorname{ad}_{\beta_{k}}^{*} \widetilde{\mu}_{k+n-1},\left(\operatorname{ad}_{\alpha_{m}}^{*} A_{m+n-1}\right)^{\sharp}\right\rangle
\end{aligned}
$$


where we have substituted $\delta E / \delta f=p^{k} \beta_{k}$ and $\delta F / \delta f=p^{m} \alpha_{m}$ and $\widetilde{\mu}_{s}(q):=\int p^{s} \mu[f] d p$.

Thus the purely dissipative moment equations are

$$
\dot{A}_{n}=\operatorname{ad}_{\gamma_{m}}^{*} A_{m+n-1} \quad \text { with } \quad \gamma_{m}:=\left(\operatorname{ad}_{\beta_{k}}^{*} \widetilde{\mu}_{k+m-1}\right)^{\sharp}
$$

If we now write the equation for $\rho:=A_{0}$ and consider only $\gamma_{0}$ and $\gamma_{1}$, we recover the following form of Darcy's law

$$
\dot{\rho}=\operatorname{ad}_{\gamma_{1}}^{*} \rho=\frac{\partial}{\partial q}\left(\rho \mu[\rho] \frac{\partial}{\partial q} \frac{\delta E}{\delta \rho}\right)
$$

where we have chosen $E=E[\rho]$ and $\widetilde{\mu}_{0}=\mu[\rho]$, so that $\gamma_{1}=\widetilde{\mu}_{0} \partial_{q} \beta_{0}$.

\subsection{Special cases}

Two interesting cases may be considered at this point. In the first case one makes Kandrup's choice in (1) for the mobility at the kinetic level $\mu[f]=f$, so that Darcy's law is written as

$$
\dot{\rho}=\frac{\partial}{\partial q}\left(\rho^{2} \frac{\partial}{\partial q} \frac{\delta E}{\delta \rho}\right) .
$$

Kandrup's case applies to the dissipatively induced instability of galactic dynamics [13]. The previous equation is the Darcy law description of this type of instability. In the second case, one considers the mobility $\mu[\rho]$ as a functional of $\rho$ (a number), leading to the equation

$$
\dot{\rho}=\mu \frac{\partial}{\partial q}\left(\rho \frac{\partial}{\partial q} \frac{\delta E}{\delta \rho}\right)
$$

which leads to the classic energy dissipation equation, $d E / d t=-\left\langle\mu \rho\left|\frac{\partial}{\partial q} \frac{\delta E}{\delta \rho}\right|^{2}\right\rangle$.

\subsection{Summary}

This section has provided a consistent derivation of Darcy's law from first principles in kinetic theory, obtained by inserting dissipative terms into the Vlasov equation which respect the geometric nature of the system. This form of the Darcy's law has been studied and analyzed in $[9,10]$, where it has been shown to possess emergent singular solutions (clumpons), which form spontaneously and collapse together in a finite time, from any smooth confined initial condition.

\section{A dissipative Vlasov equation}

The discussion from the previous sections produces an interesting opportunity for the addition of dissipation to kinetic equations. This opportunity arises from noticing that the dissipative bracket derived here could just as well be used with any type of evolution operator. In particular, we may consider introducing our bracket to modify Hamiltonian dynamics as in the approach by Kaufman [14] and Morrisson [16]. In particular, the dissipated energy may naturally be associated with the Hamiltonian arising from the corresponding Lie-Poisson theory for the evolution of a particle distribution function $f$. Therefore, we write the total dynamics generated by any functional $F(f)$ as $\dot{F}=\{F, H\}+\{\{F, E\}\}$ where $\{\cdot, \cdot\}$ represents the Hamiltonian part of the dynamics. This gives the dissipative Vlasov equation of the form (2) with $E=H$, where $H(f)$ is the Vlasov Hamiltonian. We illustrate these ideas by computing the singular (measure-valued) solution of equation (2), which represents the reversible motion of a single particle. 
Theorem 4.1 Taking $\mu(f)$ to be an arbitrary function of the smoothed distribution $\bar{f}=K * f$ for some kernel $K$ allows for single particle solutions $f=\sum_{i=1}^{N} w_{i} \delta\left(q-Q_{i}(t)\right) \delta\left(p-P_{i}(t)\right)$. The single particle dynamics is governed by canonical equations with Hamiltonian given by

$$
\mathcal{H}=\left(\frac{\delta H}{\delta f}-\left[\mu(f), \frac{\delta H}{\delta f}\right]\right)_{(q, p)=\left(Q_{i}(t), P_{i}(t)\right)}
$$

Proof. Let us write the equation of motion (2) in the following compact form

$$
\frac{\partial f}{\partial t}=-[f, \mathcal{H}], \quad \text { with } \quad \mathcal{H}:=\left(\frac{\delta H}{\delta f}-\left[\mu(f), \frac{\delta H}{\delta f}\right]\right)
$$

and substitute the single particle solution ansatz $f(q, p, t)=\sum_{i} w_{i} \delta\left(q-Q_{i}(t)\right) \delta\left(p-P_{i}(t)\right)$. Now take the pairing with a phase space function $\phi$ and write $\langle\phi, \dot{f}\rangle=-\langle[\phi, \mathcal{H}], f\rangle$. Evaluating on the delta functions proves the theorem.

Remark 3 The quantity - $[\mu(f), \delta H / \delta f]$ plays the role of a Hamiltonian for the advective dissipation process by coadjoint motion. This Hamiltonian is constructed from the momentum map $J$ defined by the $\star$ operation (Poisson bracket). That is, $J_{h}(f, g)=\left\langle g,-£_{X_{h}} f\right\rangle=\langle g,[h, f]\rangle=\langle h,[f, g]\rangle=\langle h, f \star g\rangle$.

\section{Discussion and Conclusions}

This paper has developed a new symplectic variational approach for modeling dissipation in kinetic equations based upon a double bracket structure in phase space. We focused our approach on the Vlasov example and found that the Vlasov case allows single-particle solutions, provided the mobility in the dissipation is a functional of the phase space distribution function. Moreover, we have shown how this approach recovers a nonlocal form of Darcy's law by using the Kupershmidt-Manin structure for kinetic moments. In general, it is also possible to extend our theory to the evolution of an arbitrary geometric quantity defined on any smooth manifold [12]. For example, the restriction of the geometric formalism for symplectic motion considered here to cotangent lifts of diffeomorphisms recovers the corresponding results for fluid momentum. One may also extend the present phase space treatment to include an additional set of dimensions corresponding to statistical internal degrees of freedom (order parameters, or orientation dependence) carried by the microscopic particles, rather than requiring them to be point particles. This is a standard approach in condensed matter theory, for example in liquid crystals, see, e.g., [2,3].

Remark 4 Being a special case of the dissipative bracket (3) presented in this paper, Kandrup's double bracket in (1) also possesses the Casimirs found in Proposition 2.2. However, the evolution under Kandrup's double bracket does not allow single particle solutions.

Remark 5 Had we chosen variations of the form $\delta f=-£_{X_{h}(\phi)} f=-[f, h(\phi)]$ with $h(\phi)=\mu(f) \star \phi$ $=[\mu(f), \phi]$ and followed the same steps as those in deriving (3), we would have obtained a different dissipative double bracket. It would have the same form as (3), but with $\mu(f) \leftrightarrow f$ switched in the corresponding entries. These two choices have different thermodynamic implications. In particular, the calculation in the proof of Proposition 2.3 would give entropy dynamics of the form

$$
\frac{d S}{d t}=\{\{S, E\}\}=-\left\langle\left[f, \frac{\delta E}{\delta f}\right],[\mu(f), \log f]\right\rangle=-\left\langle\frac{\mu(f)}{f},\left[f,\left[f, \frac{\delta E}{\delta f}\right]\right]\right\rangle \neq 0 .
$$

For entropy increase, this alternative variational approach would require $\mu(f)$ and $E(f)$ to satisfy an additional condition (e.g., $\mu(f) / f$ and $\delta E / \delta f$ functionally related). However, the Vlasov dissipation induced in this case would not allow the reversible single-particle solutions, because of the loss of information associated with entropy increase. 
Acknowledgements DDH and VP were partially supported by NSF grant NSF-DMS-05377891. DDH was also partially supported by the US Department of Energy, Office of Science, Applied Mathematical Research and the Royal Society Wolfson Research Merit Award. VP is grateful for the support of the Humboldt foundation and the hospitality of the Institute for Theoretical Physics, University of Cologne where this project was completed. We would also like to thank the European Science Foundation for partial support through the MISGAM program. Finally, we thank C. Josserand for helpful discussions.

\section{References}

[1] A. Bloch, P. S. Krishnaprasad, J. E. Marsden, T. S. Ratiu, The Euler-Poincaré equations and double bracket dissipation, Comm. Math. Phys. 175 (1996) 1-42.

[2] S. Chandrasekhar, Liquid Crystals, 2nd edn. Cambridge University Press, Cambridge, 1992.

[3] P. G. de Gennes and J. Prost, The Physics of Liquid Crystals, 2nd edn. Oxford University Press, Oxford, 1993.

[4] A. D. Fokker, Die mittlere Energie rotierender elektrischer Dipole im Strahlungsfeld, Annalen der Physik 43 (1914) 810-820 ; A. N. Kolmogorov, Über die analytischen Methoden in der Wahrscheinlichkeitsrechnung, Math. Ann. 104 (1931) 415-458

[5] J. Gibbons, Collisionless Boltzmann equations and integrable moment equations. Phys. D 3 (1981) 503-511.

[6] J. Gibbons, D. D. Holm, C. Tronci, Singular solutions for geodesic flows of Vlasov moments, Proceedings of the MSRI workshop "Probability, geometry and integrable systems", in celebration of Henry McKean's $75^{\text {th }}$ birthday, Cambridge University Press, Cambridge, 2007 (in press, also at arXiv:nlin/0603060

[7] J. Gibbons, D. D. Holm, C. Tronci, Vlasov moments, integrable systems and singular solutions, Phys. Lett. A, submitted (also at arXiv:0705.3603)

[8] T. L. Gilbert, A Lagrangian formulation of gyromagnetic equation of the magnetization field, Phys. Rev., 100 (1955) $1243-1255$

[9] D. D. Holm and V. Putkaradze, Aggregation of finite-size particles with variable mobility, Phys Rev Lett, 95 (2005) 226-106.

[10] D. D. Holm and V. Putkaradze, Formation of clumps and patches in self-aggregation of finite size particles, Physica D, 220 (2006) 183-196.

[11] D. D. Holm and V. Putkaradze, Interaction of particles with noncentral potential: gradient flows and singular solutions for evolution of geometric continuum quantities, Physica D, to appear (2007).

[12] D. D. Holm and V. Putkaradze, C. Tronci, Geometric evolution equations for order parameters, Physica D, submitted.

[13] H. E. Kandrup, The secular instability of axisymmetric collisionless star cluster. Astrophy. J. 380 (1991) 511-514.

[14] A. N. Kaufman, Dissipative Hamiltonian systems: a unifying principle, Phys. Lett. A, 100 (1984) 419-422.

[15] B. A. Kupershmidt, Ju. I. Manin, Long wave equations with a free surface. II. The Hamiltonian structure and the higher equations. Funktsional. Anal. i Prilozhen. 12 (1978) 25-37

[16] P. J. Morrison, Bracket formulation for irreversible classical fields, Phys. Lett. A, 100 (1984) 423-427.

[17] F. Otto, The geometry of dissipative evolution equations: the porous medium equation. Comm. Partial Differential Equations 26 (2001) 101-174

[18] A. A. Vlasov, On the kinetic theory of an assembly of particles with collective interaction. J. Phys. (USSR) 9 (1945) 25-40; A. A. Vlasov, Many-particle theory and its application to plasma. Gordon and Breach, New York, 1961 